\title{
Treinamento Odontológico Imersivo por meio de Realidade Virtual
}

\author{
Romero Tori $^{1}$, Gustavo Ziyu Wang ${ }^{1}$, Lucas Henna Sallaberry ${ }^{1}$, Allan Amaral Tori ${ }^{2}$ \\ Elen Collaço de Oliveira ${ }^{1}$, Maria Ap. de A. M. Machado ${ }^{2}$ \\ ${ }^{1}$ Escola Politécnica \\ Universidade de São Paulo (USP) - São Paulo - SP - Brasil \\ ${ }^{2}$ Faculdade de Odontologia de Bauru \\ Universidade de São Paulo (USP) - Bauru - SP - Brasil \\ \{gustavo.ziyu, lhsallaberry, allant22, elencollaco\}@gmail.com \\ torieacm.org, mmachado@fob.usp.br
}

\begin{abstract}
Training invasive medical procedures at the pre-clinical development is a considerable educational challenge. The traditional approach, with animals and corpses, presents both ethical and practical issues. So, the use of virtual reality $(V R)$, which enables low cost realistic simulations, repeatability and real time feedback, is a promising solution. Such ascertainment motivated the development of the virtual environment VIDA Odonto 1, which resorts to VR technologies, realistic modeling of patient and tridimensional immersive interaction. The developed prototype was tested and evaluated by professionals with clinical experience and beginners, demonstrating its viability to be a relevant educational resource in dentistry courses.
\end{abstract}

Resumo. O treinamento de procedimentos médicos invasivos na etapa préclínica é um grande desafio educacional. A abordagem tradicional, com animais e cadáveres, apresenta problemas éticos e práticos. Uma solução promissora é a utilização de realidade virtual $(R V)$, que permite simulações realistas de baixo custo, repetibilidade e feedback em tempo real. Tal constatação motivou o desenvolvimento do ambiente VIDA Odonto, o qual utiliza tecnologias avançadas de $R V$, modelagem realista do paciente e interação imersiva tridimensional. O protótipo desenvolvido foi testado e avaliado por profissionais com experiência na prática clínica e principiantes, demonstrando a sua viabilidade de se tornar um recurso educacional relevante em cursos de odontologia.

\section{Introdução}

Treinar futuros médicos, enfermeiros ou dentistas traz grandes desafios por envolver seres humanos, pela necessidade de infra-estrutura de alto custo e pela complexidade e delicadeza dos procedimentos. Para o ensino de anatomia, a base de qualquer formação na área de saúde que envolve a capacitação de profissionais que atenderão pacientes, costumam ser utilizados diversos meios, tais como laboratórios que possibilitam a manipulação de corpos humanos e de animais, modelos e peças anatômicas, vídeos, imagens e multimídia [Battulga et al. 2012]. Existem diversos problemas nessa abordagem tradicional. A manipulação de organismos apresenta questões éticas, morais e culturais. Além disso, 
V Congresso Brasileiro de Informática na Educação (CBIE 2016)

Anais do XXVII Simpósio Brasileiro de Informática na Educação (SBIE 2016)

restos de corpos humanos não fornecem respostas fisiológicas aos estudantes que os manipulam, diminuindo a percepção de realismo [McLachlan et al. 2004]. A maneira como a morte é apresentada aos alunos é um problema adicional. Estudantes expostos a cadáveres apresentam uma maior frequência de alterações psicológicas e mentais, de acordo com estudos realizados nos Estados Unidos e Austrália [Hancock et al. 1998, Gustavson 1988]. Eles podem inclusive desenvolver atitudes inapropriadas para lidarem com o estresse causado por essas aulas [Finkelstein and Mathers 1990]. Apesar desses problemas, o aprendizado baseado nas multimídias atuais, como livros e tutorias virtuais, ainda não obteve êxito em substituir a dissecação. Uma das principais críticas dos estudantes de anatomia, nesse caso, é a falta de percepção de profundidade [Azer and Eizenberg 2007]. Treinamentos auxiliados por dispositivos de realidade virtual e reconhecimento de gestos, por outro lado, possibilitam realismo, percepção de imersão [Xu 2006, Vora et al. 2002], por meio da visão estereoscópica, e repetibilidade dos procedimentos, com baixo custo e sem os problemas éticos e logísticos presentes em formas tradicionais de treinamento.

A partir dos resultados obtidos no projeto VIDA (Virtual Interactive Distancelearning on Anatomy) [Melo et al. 2011, Tori et al. 2009a, Tori et al. 2009b], em que foram desenvolvidas interfaces interativas tridimensionais imersivas, e da experiência adquirida com o projeto Vimet Odonto [Corrêa 2015], que gerou uma interface háptica para simulação de anestesia do nervo alveolar inferior, foi desenvolvido e avaliado o protótipo de uma ferramenta imersiva, baseada em interação tridimensional, para treinamento de anestesia odontológica, denominada VIDA Odonto. Essa ferramenta de baixo-custo, quando comparada aos instrumentos educacionais convencionais citados, pode ser utilizada presencialmente ou a distância, com capacete HMD (Head-Mounted Display) ou com adaptadores de baixo-custo para smartphones. A ferramenta também avalia automaticamente a precisão do procedimento realizado pelo treinando e permite a gravação para futura avaliação pelo professor ou pelo próprio aluno. Tal recurso ainda pode ser usado na preparação de vídeos didáticos imersivos.

\section{Cenário e objetivos}

Os estudantes de odontologia precisam passar por treinamentos pré-clínicos sucessivos antes de executarem pela primeira vez o procedimento de anestesia em uma pessoa para a execução de um procedimento clínico. A exemplo de pilotos de avião, e de outras profissões que envolvem a integridade e saúde de seres humanos, não é tolerável que uma pessoa não adequadamente treinada, avaliada e aprovada seja liberada para atuação profissional. No caso dos pilotos já é imperativo o uso de simuladores de voo. Em um futuro próximo é provável que estudantes da área de saúde também tenham o treinamento em simuladores incluídos em suas grades curriculares.

Numa parceria entre o Laboratório de Tecnologias Interativas (Interlab) do Depto de Engenharia de Computação da Escola Politécnica de São Paulo e a Faculdade de Odontologia de Bauru da Universidade de São Paulo, está sendo desenvolvido pelos autores um ambiente imersivo para treinamento de procedimentos odontológicos, que foi denominado "Laboratório de Simulação e Treinamento"(LaSIT), do qual a ferramenta VIDA Odonto fará parte. O primeiro procedimento a ser testado com essa ferramenta é o de anestesia do nervo alveolar inferior. Posteriormente o ambiente VIDA Odonto será expandido a fim de possibilitar treinamentos de outros procedimentos da rotina da clínica odontológica, que poderão englobar desde a obtenção e análise de imagens de radiografias 
V Congresso Brasileiro de Informática na Educação (CBIE 2016)

Anais do XXVII Simpósio Brasileiro de Informática na Educação (SBIE 2016)

até à esterilização e organização de instrumentais, passando por interações com o paciente e suas reações emocionais e físicas. Por esse motivo está sendo modelado em detalhes um consultório dentário (Figura 1a) e um paciente de 10 anos de idade, em corpo inteiro e com alto detalhamento interno das estruturas anatômicas da boca incluindo maxilar e mandíbula (Figura 1b).
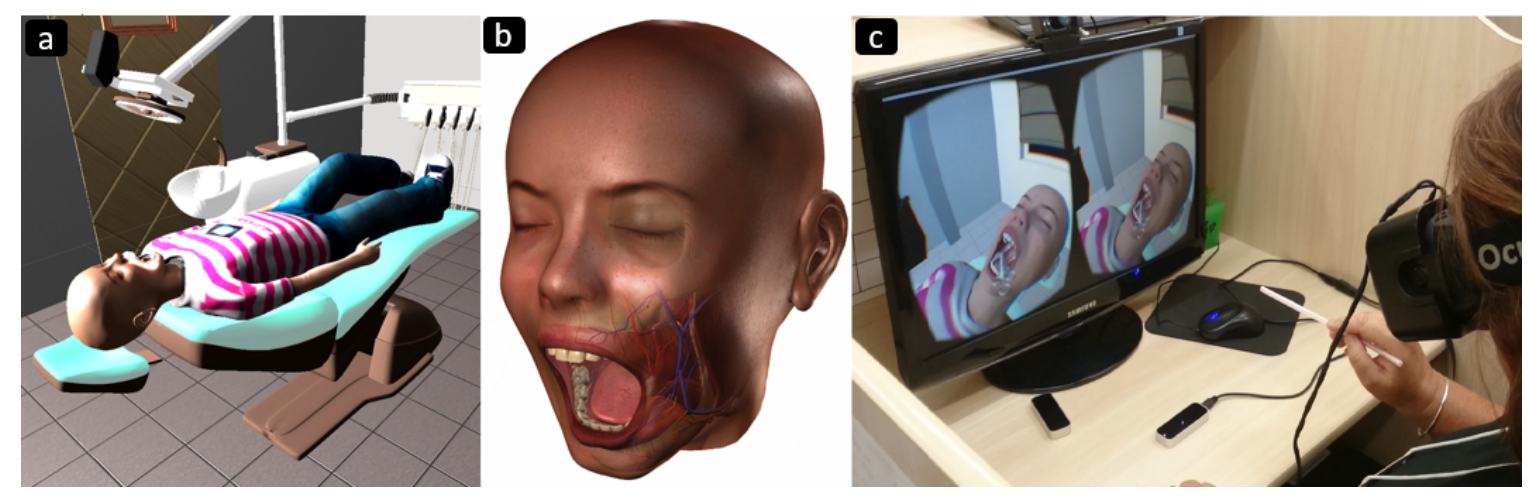

Figura 1. Consultório odontológico virtual, modelo 3D da cabeça e especialista aplicando uma injeção no VIDA Odonto

A ferramenta está sendo projetada de forma a prover ao estudante de odontologia nas etapas iniciais de sua formação pré-clínica, sensações de presença [Biocca et al. 2001, Tori 2010] e imersão, isto é, provocar no usuário a sensação de estar envolvido com o ambiente odontológico e com pacientes virtuais e poder interagir como se estivesse em situações próximas àquelas que ocorrem no aprendizado em um laboratório real. Laboratórios virtuais imersivos de alta tecnologia, baseados em CAVE, capacetes HMD profissionais, luvas de dados e outros dispositivos acoplados ao corpo [Tori et al. 2009a], atenderiam tais requisitos, mas por apresentarem altos custos (alguns não são produzidos no Brasil exigindo importação e são pouco flexíveis), inviabilizariam o uso em alta escala.

A hipótese inicial do VIDA Odonto é que, com técnicas de reconhecimento de gestos e de imersão em ambiente tridimensional, é viável a criação de um ambiente de baixo custo que possibilite ao estudante de odontologia treinar numa nas etapas de formação pré- e pós-clínica procedimentos de anestesia de forma intuitiva, natural, com baixa sobrecarga cognitiva e alta percepção de presença, como se estivesse realizando-os em pacientes reais.

O experimento apresentado neste artigo teve por objetivo desenvolver e testar um protótipo imersivo de um consultório odontológico dotado de funcionalidades básicas para a realização de um procedimento clínico fundamental para a prática clínica, a anestesia. O protótipo apresentado permite ao operador realizar o movimento inicial da seringa, avaliação de sua precisão e gravação do procedimento. O teste da primeira etapa do movimento (aproximação da seringa e posicionamento sobre o ponto de inserção) foi realizado por profissionais e professores experientes, bem como por alunos iniciantes de odontologia e de outros cursos (simulando alunos sem qualquer experiência). Os movimentos foram gravados e avaliados, quanto a sua precisão, pelo módulo de avaliação automatizada. Foi também avaliado o grau de realismo no espaço virtual percebido pelos participantes durante a realização do procedimento. 
V Congresso Brasileiro de Informática na Educação (CBIE 2016)

Anais do XXVII Simpósio Brasileiro de Informática na Educação (SBIE 2016)

\section{Interações em Ambientes Tridimensionais}

$\mathrm{A}$ interação em $\mathrm{AV}$ se dá por meio de operações de manipulação de objetos com vistas a atingir objetivos específicos. Para cada operação realizada o AV deve produzir um feedback adequado. Segundo Pinho e Rebelo (2004) [Pinho and Rebelo 2004] são três os elementos de uma interface homem-máquina: dispositivo de entrada, que captura ações ou estímulos gerados pelos usuários; função de transferência, que mapeia a ação capturada para um elemento ou elementos controlados pelo sistema; e dispositivo de saída, que envia uma resposta à ação executada.

As interações devem ser projetadas levando em consideração características e limitações dos dispositivos de interface, para que sejam adequadas ao cenário e públicoalvo a que se destinam. Segundo Bowman et al. (2001) [Bowman et al. 2001], as operações podem ser agrupadas em três categorias: navegação, seleção/manipulação e controle do sistema. A navegação consiste no movimento do usuário dentro do AV, a seleção/manipulação se refere à escolha de um objeto virtual e à modificação de suas características, e o controle do sistema define comandos específicos para modificar o estado do sistema.

Em ambientes tridimensionais voltados para a educação médica, um cuidado especial deve ser tomado com a precisão na modelagem do ambiente, equipamentos e, principalmente, das partes orgânicas que serão manipuladas pelo sistema. Por se destinar a uso em educação a distância deve também ser levada em conta a diversidade de equipamentos, plataformas e configurações que os usuários remotos terão. Para atender às necessidades expostas foram incluídos os seguintes requisitos: a disponibilidade para uso de dispositivos de entrada e saída de baixo custo (requisito de sistema) e a naturalidade do processo de interação (requisito de interface).

\section{Sistemas Relacionados}

O projeto VIDA (Virtual Interactive Distance-learning on Anatomy) [Melo et al. 2011, Tori et al. 2009a, Tori et al. 2009b], desenvolveu um novo paradigma de interação, denominado holográfico, cujo princípio era o de dar ao usuário a sensação de estar manipulando os objetos anatômicos virtuais com as mãos livres, como se fossem holografias e possuíssem volume. Por ser destinado a cursos a distância e ter como requisito o baixocusto, a melhor solução na época foram os óculos de anáglifo. Toda a interação do VIDA é baseada em gestos naturais, que foram definidos a partir de estudos etnográficos realizados com potenciais usuários. Para evitar menus, que quebrariam a ilusão do efeito holográfico, comandos de sistema são acionados por voz. Os gestos deveriam ser necessariamente naturais, como se os objetos fossem manipulados fisicamente, nunca gestos de comando. Os princípios de interface do ambiente VIDA foram transferidos para o VIDA Odonto, com a diferença que este passa a usar tecnologia de software e equipamentos de imersão de realidade virtual mais atuais, que melhor atendam hoje aos requisitos do sistema.

O sistema Vimet Odonto [Corrêa 2015] teve como objetivo a simulação da anestesia do nervo alveolar inferior para treinamento odontológico. Diferentemente do ambiente objeto deste trabalho, cujo foco principal é a interação imersiva, o foco do Vimet Odonto foi a interação háptica. Por utilizar tecnologias Java e Java $3 D$ não foi possível aproveitar o que já foi desenvolvido no Vimet para incorporação no VIDA Odonto. Pretende- 
se, no entanto, utilizar todo o conhecimento gerado pelo projeto Vimet Odonto para incorporação de um futuro módulo de interface háptica no sistema.

Um simulador de realidade virtual para treinamento da aplicação de anestesia para bloqueio do nervo alveolar inferior foi desenvolvido e utilizado em faculdades de odontologia na Escócia para familiarizar alunos com o procedimento [Poyade et al. 2014]. O simulador conta com um modelo anatomicamente preciso de uma cabeça e pescoço humano, incluindo os músculos, nervos, ossos e vasos sanguíneos. Utiliza-se uma interface háptica para o retorno de força da seringa penetrando os diferentes tecidos. Como confirmado por especialistas da área de odontologia, o procedimento a ser treinado por meio do VIDA Odonto apresenta diferenças quando aplicado em um adulto (como no trabalho citado) e em uma criança, justificando a necessidade de se criar ambos os modelos para treinos. Outros pontos levantados no trabalho de Poyade et. al (2014) foram a limitação do alcance do aparelho háptico e utilização da ferramenta padrão deste aparelho, que foge muito ao usado pelos dentistas, a seringa, para realizar o treinamento. Estas limitações podem ser amenizadas pela utilização de uma tecnologia de rastreamento de objetos, como a utilizada neste trabalho, permitindo um espaço de trabalho mais amplo, além de aceitar diferentes instrumentos para rastreamento.

\section{VIDA Odonto}

O sistema VIDA Odonto utiliza realidade virtual imersiva para oferecer um ambiente virtual para treinamento de procedimentos odontológicos, aplicando os conceitos de manipulação direta e interface holográfica do ambiente VIDA. Sendo o sistema um ambiente de aprendizagem que terá constante expansão, nesta seção será apresentada a arquitetura projetada para o sistema expandido. Seu desenvolvimento é interdisciplinar, acompanhado por especialistas da área de odontologia, design e engenharia. O protótipo implementado do módulo de simulação (seção 6), em relação a outros sistemas existentes (seção 4), apresenta os seguintes diferenciais:

- interface holográfica de manipulação direta (entenda-se aqui "paradigma holográfico"como sendo a simulação de se interagir com a projeção de objetos virtuais volumosos, como se esses estivessem ao alcance das mãos do interator, sem intermediação, seja por apontadores ou elementos de interface);

- imersão; possibilidade de se movimentar pelo consultório virtual e se posicionar como se estivesse ao lado da cadeira do paciente;

- avaliação automática da precisão do movimento;

- gravação do experimento para posterior visualização imersiva.

\subsection{Avaliação Automática}

Um dos recursos inovadores do VIDA Odonto é a possibilidade de avaliação automática do desempenho do treinando durante a realização do procedimento. Para tanto o sistema prevê a gravação de todo o trajeto vetorial da agulha, que pode ser acionado pelo professor durante o treinamento, para posterior análise automatizada. Em versões futuras essa análise poderá ser realizada em tempo real. Uma vez tendo-se o registro do procedimento ideal, realizado por um especialista, pode-se compará-lo à trajetória desenvolvida pelo treinando e obter uma nota, e eventuais críticas, do que foi exercitado. 
V Congresso Brasileiro de Informática na Educação (CBIE 2016)

Anais do XXVII Simpósio Brasileiro de Informática na Educação (SBIE 2016)

A partir de informações colhidas com especialistas, sabe-se que os dois principais aspectos a avaliar no processo de aproximação e posicionamento da agulha são: proximidade da agulha do ponto alvo na boca e ângulo sólido de posicionamento da agulha (Figura $2 b$ ). Para se chegar a uma nota entre 0 e 1 são multiplicados três termos. O primeiro recebe um valor 0 ou 1 ( 0 : erro grave, como por exemplo atingir acidentalmente alguma parte do rosto do aluno ou não chegar ao ponto de introdução da agulha). O segundo termo se refere à inclinação do posicionamento da agulha em relação à direção considerada de referência. Esse termo depende do cosseno do ângulo formado pela direção da agulha posicionada pelo aluno em relação à direção de referência, elevado a um coeficiente de calibração. Esse coeficiente definirá a velocidade com que a nota se aproximará de zero à medida em que o A partir de informações colhidas com especialistas, sabe-se que os dois principais aspectos a avaliar no processo de aproximação e posicionamento da agulha são: proximidade da agulha do ponto alvo na boca e ângulo sólido de posicionamento da agulha (Figura 2b). Para se chegar a uma nota entre 0 e 1 são multiplicados três termos. O primeiro recebe um valor 0 ou 1 ( 0 : erro grave, como por exemplo atingir acidentalmente alguma parte do rosto do aluno ou não chegar ao ponto de introdução da agulha). O segundo termo se refere à inclinação do posicionamento da agulha em relação à direção considerada de referência. Esse termo depende do cosseno do ângulo formado pela direção da agulha posicionada pelo aluno em relação à direção de referência, elevado a um coeficiente de calibração. Esse coeficiente definirá a velocidade com que a nota se aproximará de zero à medida em que o ângulo do erro aumenta. O terceiro termo contribui com o erro de distanciamento do ponto alvo, obtido pelo complemento da divisão, por um coeficiente de calibração linear entre o ponto de referência e o ponto atingido pelo aluno.
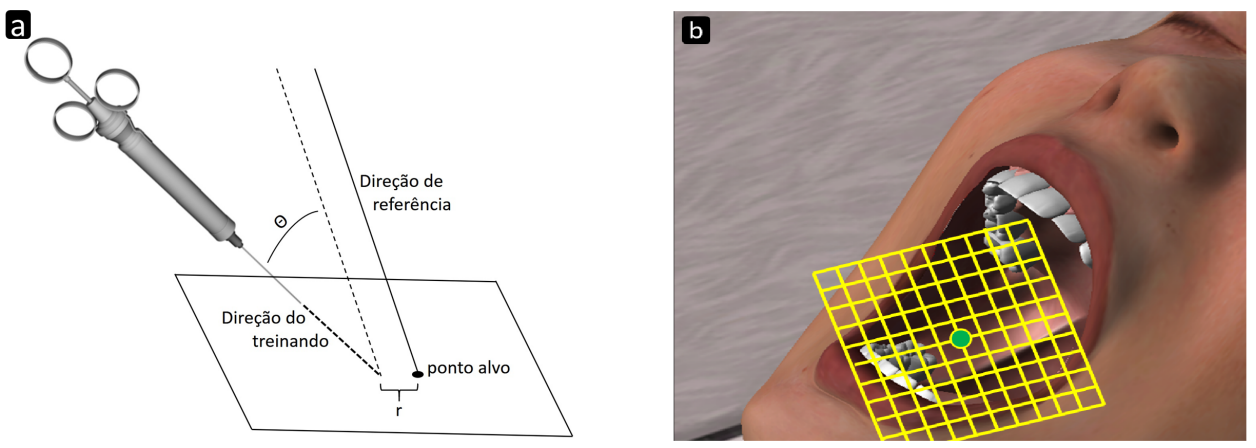

Figura 2. Parâmetros usados na avaliação

A partir do exposto chegou-se à seguinte formulação para a nota do procedimento:

$$
\begin{gathered}
N_{F}=N_{\theta} * N_{r} * N_{e} * 10 \\
N_{\theta}=\cos ^{a} \theta \\
N_{r}=1-\frac{r}{d} ; r<=d
\end{gathered}
$$

e $\theta$ o ângulo em relação ao referencial utilizado, $a$ o coeficiente de calibração angular, $r$ a distância do ponto aplicado em relação ao referencial utilizado, $d$ o coeficiente de calibração linear. $N_{e}=0$ no caso do usuário atingir acidentalmente a pele com a ponta 
V Congresso Brasileiro de Informática na Educação (CBIE 2016)

Anais do XXVII Simpósio Brasileiro de Informática na Educação (SBIE 2016)

da seringa ou interromper o procedimento antes de atingir a região alvo. Caso contrário, $N_{e}=1$.

\subsection{Design e Modelagem 3D}

O propósito do Design neste experimento foi pesquisar, modelar e texturizar o objeto 3D virtual da estrutura óssea do crânio e das camadas de tecidos da face, presentes na região do nervo alveolar inferior, equivalente a apresentada pelo público infantil, na faixa etária dos 07 aos 12 anos, considerando as características físicas e reais apresentadas por cada tecido (tecidos moles, músculos, nervos). A importância dessa modelagem é simular de forma realística a anatomia da face, com a visualização das camadas de tecidos presentes na região citada, proporcionando um ambiente virtual rico para imersão do aluno, durante a realização da técnica de bloqueio dessa região da boca acessado via computador pelo aluno de graduação em odontologia para seu treinamento.

O princípio da metodologia aplicada na modelagem 3D foi o levantamento de modelos anatômicos das estruturas que seriam modeladas, desde aulas e livros de anatomia, imagens da internet e principalmente ressonâncias magnéticas. Em seguida, o crânio em 3D, que foi o ponto de partida do processo de modelagem 3D no software Autodesk Maya 2013, foi desenvolvido com o auxílio de esboços em papel. Com o crânio e arcada dentária em 3D finalizados, passou-se para a modelagem dos músculos, com foco na região da boca. As modelagens das veias, artérias e nervos, foram feitas em seguida, permitindo finalizar o processo com as camadas da pele e gengiva. Todas as modelagens foram feitas com um número reduzido de polígonos, para não sobrecarregar o sistema.

Após as modelagens construídas e finalizadas, os estudos concentraram-se no desenvolvimento das texturas superficiais dos tecidos. As texturas são imagens em formato JPEG, e foram desenvolvidas no software Adobe Photoshop CS6. Para se criar a textura da parte óssea, foi necessário tirar fotos de um crânio real em 5 posições diferentes, para que depois pudesse ser feito uma montagem com todas elas, gerando uma única imagem ou textura. Para o resto da modelagem foi usada a técnica de pintura digital e montagens de imagens livres e permitidas encontradas na internet. Uma clínica com alguns objetos fundamentais foi desenvolvida, como maca odontológica, pia, janela, paredes e chão. Um corpo de criança também foi criado de forma simplificada, ou seja, com poucos polígonos.

\subsection{Prova de conceito}

A prova de conceito foi desenvolvida utilizando a plataforma Unity 4.6, na qual foram acrescentadas as bibliotecas do Oculus Rift DK2 e Leap Motion. A figura 1b apresenta o modelo do consultório odontológico com o paciente visto de frente.

O Leap Motion foi utilizado no modo de detecção de ferramentas, rastreando um objeto cilíndrico real representado no meio virtual por uma seringa. Acoplada à agulha da ferramenta virtual, há um detector de colisões que identifica a região da cabeça na qual a seringa realiza contato. Os movimentos da seringa foram limitados para que seu corpo não pudesse atravessar nenhum tecido, desta forma não interferindo na sensação de imersão.

Sendo o objetivo da simulação a aplicação de anestesia no nervo alveolar inferior, foi implementado no ponto de injeção um plano que, por meio de um script, calcula o ângulo de inclinação da seringa durante a aplicação (Figura 2b), para extração 
V Congresso Brasileiro de Informática na Educação (CBIE 2016)

Anais do XXVII Simpósio Brasileiro de Informática na Educação (SBIE 2016)

dos parâmtetros utilizados pelo módulo de avaliação automática (Figura 2a). Com as informações do rastreamento do Leap Motion, obtém-se também a posição no espaço virtual tridimensional da agulha da seringa em função do tempo.

O programa exporta no final de cada simulação um arquivo texto com as informações previamente detalhadas, utilizando a persistência de dados da linguagem Csharp, com a qual foram escritos todos os códigos da simulação. Por fim, foram implementados comandos para escolher qual o tipo de visão desejada: por HMD ou pelo monitor. Para efeito de prova de conceito foram utilizados, para a avaliação automática do procedimento, os coeficientes $a=2$ e $d=4$ (a distância $\mathrm{r}$ do ponto de colocação da agulha em relação ao alvo de referência é medida em centímetros).

\section{Resultados}

Utilizando como referência a aplicação da injeção por uma profissional da área de odontologia (Figura 1c), foram analisadas as aplicações da injeção com e sem o dispositivo HMD para cada participante.

Foram avaliados 14 participantes, sendo 5 deles especialistas e 9 não especialistas. A Figura 3 apresenta a comparação entre as notas concedidas para os participantes ao realizar o experimento com e sem o dispositivo HMD. É possível observar que houve uma melhora no desempenho da aplicação da injeção com o uso do dispositivo HMD. Com efeito, nota-se que 11 dos participantes obtiveram uma nota maior.

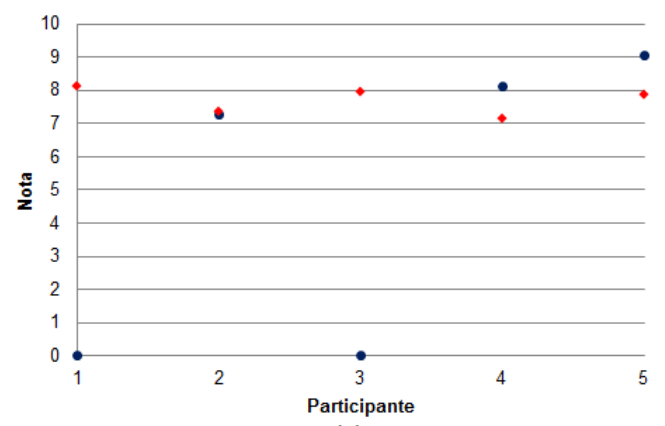

(a)

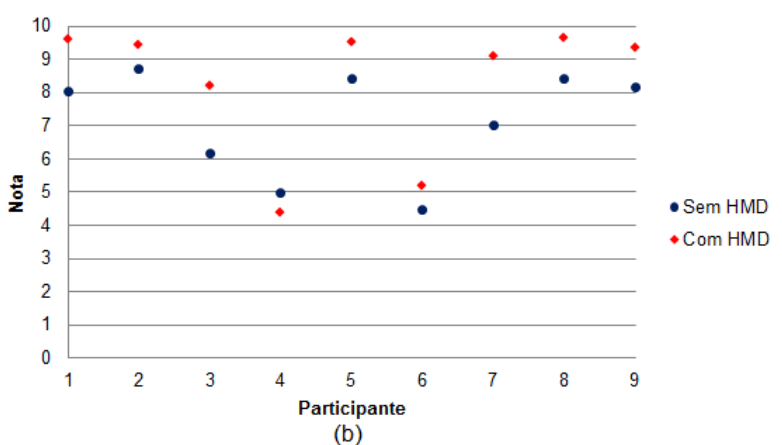

(b)

Figura 3. Notas atribuídas. (a) Especialistas. (b) Não especialistas.

Além disso, os participantes, imediatamente após o experimento, preencheram um questionário contendo as seguintes perguntas dissertativas:

- Apresente as dificuldades e os problemas encontrados.

- Apresente os pontos fortes do sistema.

- Sugestões.

- Faça suas considerações gerais sobre o sistema.

Foi possível observar dessas respostas que um dos pontos mais levantados pelos participantes foram as dificuldades de se usar o sensor de movimento (Leap Motion) juntamente com o instrumento cilíndrico. Observou-se durante o experimento que a seringa virtual apresentava instabilidade por dois motivos : a presença de pontos cegos/ângulos de difícil detecção que tornavam a seringa instável até quando o participante não tremia e a leveza do objeto cilíndrico quando comparado à seringa. A utilização do instrumento 
V Congresso Brasileiro de Informática na Educação (CBIE 2016)

Anais do XXVII Simpósio Brasileiro de Informática na Educação (SBIE 2016)

cilíndrico de forma precisa foi considerada um desafio de difícil adaptação, porém aqueles que já conheciam a tecnologia do sensor demonstraram um domínio e precisão muito maior, indicando que, após um tempo de familiarização (que não foi possível durante o experimento), é provável que o participante tenha muito mais facilidade com o equipamento. Apesar disso, entre os participantes mais novos (nascidos em 96 e 97) e que não conheciam o sensor, foram feitos comentários positivos quanto à precisão do sensor por três dos seis participantes, sendo estes os únicos comentários positivos sobre o sensor registrados após o experimento. Dois deles, porém, fizeram comentários negativos e o outro se manteve neutro. Ainda assim, esses dados podem demonstrar uma maior aceitação de tecnologias mais novas por alunos jovens (mais próximos da idade alvo deste treinamento) quando comparado a participantes mais velhos.

Outro ponto levantado foi a falta de resistência na perfuração do tecido. Esse tipo de comentário teve origem dos participantes especialistas da área de odontologia que conhecem o projeto Vimet Odonto e já conheciam aparelho de retorno de força háptico, relatando que o retorno de força é bastante relevante para este tipo de experimento.

Foram feitos elogios e críticas tanto ao modelo do paciente quanto ao consultório. Em especial, foi observado pelos especialistas que o paciente não apresentava alguns pontos de referência para a aplicação da anestesia. No geral, o ambiente do consultório foi bem aceito e grande parte dos participantes comentou ter a sensação de imersão. Também foram observados comentários sobre alguns pontos referentes à importância de se ter um simulador para esse tipo de procedimento, a relevância de se criar uma alternativa ao método convencional e a conveniência do auto treinamento que pode ser realizado.

\section{Conclusão}

Os resultados deste experimento demonstraram a viabilidade de se utilizar tecnologias de realidade virtual para o treinamento de estudantes da área de odontologia na etapa pré-clínica. Observou-se que grande parte dos participantes comentaram ter a sensação de imersão. Esse é um poderoso indicativo para, em um próximo experimento, analisar as questões de "presença"do sistema, a fim de verificar o quanto o participante falha em perceber a mediação da tecnologia utilizada durante o processo de interação.

Os próximos passos do ambiente VIDA Odonto incluem: inclusão de interface háptica, adaptada a uma seringa idêntica à utilizada pelos profissionais durante o procedimento e anestesia; desenvolvimento do módulo do professor, que incluirá o compartilhamento do espaço virtual tridimensional; adaptação para smarthphones, usando adaptadores de baixo custo, como o Google cardboard; estudos com novos dispositivos, como o Hololens da Microsoft; inclusão de um boneco palpável, devidamente registrado com o paciente virtual, possibilitando maior realismo ao treinando.

\section{Referências}

Azer, S. A. and Eizenberg, N. (2007). Do we need dissection in an integrated problembased learning medical course? perceptions of first-and second-year students. Surgical and Radiologic Anatomy, 29(2):173-180.

Battulga, B., Konishi, T., Tamura, Y., and Moriguchi, H. (2012). The effectiveness of an interactive 3-dimensional computer graphics model for medical education. Interactive journal of medical research, 1(2). 
V Congresso Brasileiro de Informática na Educação (CBIE 2016)

Anais do XXVII Simpósio Brasileiro de Informática na Educação (SBIE 2016)

Biocca, F., Harms, C., and Gregg, J. (2001). The networked minds measure of social presence: Pilot test of the factor structure and concurrent validity. In 4th annual International Workshop on Presence, Philadelphia, PA, pages 1-9.

Bowman, D. A., Kruijff, E., LaViola Jr, J. J., and Poupyrev, I. (2001). An introduction to 3-d user interface design. Presence: Teleoperators and virtual environments, 10(1):96.

Corrêa, C. G. (2015). Simulação de inserção de agulha para treinamento de procedimento de anestesia odontológica. $\mathrm{PhD}$ thesis, Universidade de São Paulo.

Finkelstein, P. and Mathers, L. H. (1990). Post-traumatic stress among medical students in the anatomy dissection laboratory. Clinical Anatomy, 3(3):219-226.

Gustavson, N. (1988). The effect of human dissection on first-year students and implications for the doctor-patient relationship. Academic Medicine, 63(1):62-4.

Hancock, D., Williams, M., and Taylor, A. (1998). Psychological impact of cadavers and prosections on physiotherapy and occupational therapy students. Australian Journal of Physiotherapy, 44(4):247-255.

McLachlan, J. C., Bligh, J., Bradley, P., and Searle, J. (2004). Teaching anatomy without cadavers. Medical education, 38(4):418-424.

Melo, C., Aceiro, J., Nunes, F., Tori, R., and Muntoreanu, I. (2011). Implementação de atlas virtual: Definição de procedimentos e ações para o ensino da assistência ao parto. VII Congresso Brasileiro de Enfermagem Obstétrica e Neonatal, pages 4291-4297.

Pinho, M. and Rebelo, I. (2004). Interação em ambientes virtuais imersivos. In : Realidade Virtual - Conceitos e Tendências, page 109.

Poyade, M., Lysakowski, A., and Anderson, P. (2014). Development of a haptic training simulation for the administration of dental anaesthesia based upon accurate anatomical data.

Tori, R. (2010). Educação sem distância - as tecnologias interativas na redução de distâncias em ensino e aprendizagem. São Paulo: Senac, 2010. 256 p.

Tori, R., Marques, F., Nakamura, R., Bernardes, J., Corrêa, C., and Tokunaga, D. (2009a). Design de interação para um atlas virtual de anatomia usando realidade aumentada e gestos. Interaction South America, 9.

Tori, R., Nunes, F. L., Gomes, V. H., and Tokunaga, D. M. (2009b). Vida: Atlas anatômico $3 \mathrm{~d}$ interativo para treinamento a distância. In XXIX Congresso da Sociedade Brasileira de Computação, volume 1.

Vora, J., Nair, S., Gramopadhye, A. K., Duchowski, A. T., Melloy, B. J., and Kanki, B. (2002). Using virtual reality technology for aircraft visual inspection training: presence and comparison studies. Applied Ergonomics, 33(6):559-570.

$\mathrm{Xu}, \mathrm{D}$. (2006). A neural network approach for hand gesture recognition in virtual reality driving training system of spg. In Pattern Recognition, 2006. ICPR 2006. 18th International Conference on, volume 3, pages 519-522. IEEE. 